\title{
Spontaneous pregnancy following post in vitro fertilization ruptured tubal ectopic pregnancy
}

\author{
Kirty Nahar $^{1 *}$, Nikita Nahar ${ }^{2}$ \\ ${ }^{1}$ Department of Obstetrics and Gynecology, Apollo Hospital International ltd., Ahmedabad, Gujarat, India
${ }^{2}$ Smt. NHL Municipal, Medical College, Ahmedabad, Gujarat, India
}

Received: 21 August 2020

Accepted: 30 September 2020

\author{
*Correspondence: \\ Dr. Kirty Nahar, \\ E-mail: naharkirty@yahoo.co.in
}

Copyright: (c) the author(s), publisher and licensee Medip Academy. This is an open-access article distributed under the terms of the Creative Commons Attribution Non-Commercial License, which permits unrestricted non-commercial use, distribution, and reproduction in any medium, provided the original work is properly cited.

\begin{abstract}
Ectopic pregnancy (EP) is a dramatic life threatening event in a woman's reproductive life, especially after a long, expensive and difficult course of treatment for infertility. EP accounts around 1-2\% of all natural conceptions, and this prevalence increases following assisted reproductive techniques, to range between $2.1 \%$ and $8.6 \%$ and it can reach up to $11 \%$ in women with tubal factors infertility history. A 32 year old female, primigravida presented at emergency department of Apollo Hospitals, Ahmedabad with complaints of amenorrhoea 2 months, severe pain abdomen associated with vomiting, difficulty in breathing and bleeding per vagina on and off. She was a case of primary infertility with polycystic ovary syndrome (PCOS) who had conceived after difficulty with in vitro fertilization (IVF), resulted in ruptured right tubal ectopic pregnancy. She underwent exploratory laparotomy followed by removal of right ectopic pregnancy, right salpingectomy and peritoneal lavage. Early diagnosis, timely intervention and prompt surgical management could save the patient's life. Later on she conceived spontaneously and had an eventful and complicated pregnancy. She presented at 35 weeks of pregnancy with preterm labour pain and underwent emergency caesarean section for fetal distress. She delivered a healthy male child and had a successful obstetric outcome. Diagnosis of ruptured tubal ectopic pregnancy is made based on patient's history, clinical acumen, serum beta human chorionic gonadotropin (hCG) levels and pelvic ultrasound. Ectopic pregnancy should be suspected in patients with an adnexal mass even in absence of risk factors. Clinicians must be alert to the fact that assisted reproductive techniques as a treatment for infertility can result into ectopic pregnancy. This case highlights the fact that patient who underwent IVF treatment resulting in ruptured tubal ectopic pregnancy can have spontaneous conception and a successful obstetric outcome.
\end{abstract}

Keywords: Ectopic pregnancy, Salpingectomy, Assited reproductive techniques, Invitro fertilization, Embryo transfer

\section{INTRODUCTION}

Ectopic pregnancy (EP) is still one of the important causes of maternal mortality. ${ }^{1}$ With the widespread and frequent use of assisted reproductive techniques (ART), the frequency of abnormally located pregnancies also rises. ${ }^{2}$

In vitro fertilization (IVF) with embryo transfer (ET) has been reported to result in a higher rate of EP than spontaneous pregnancies. Approximately $2.1-8.6 \%$ of all clinical pregnancies after IVF-ET have been reported to be ectopic compared with an ectopic pregnancy rate of $2 \%$ after natural conception. ${ }^{3-5}$

Misdiagnosis or delayed diagnosis of EP leads to such complications as severe bleeding and hypovolemic shock associated with maternal morbidity and mortality. Pelvic inflammatory disease, tubal infertility, previous pelvic surgery, and congenital uterine abnormalities have been defined as the leading factors contributing to the risk of EP in women with infertility. ${ }^{6-8}$ The number and quality of transferred embryos, controlled ovarian stimulation (COS) 
regimens, laboratory conditions, and transfer techniques have been offered as possible contributors to the occurrence of EP after IVF-embryo transfer (ET). ${ }^{9}$

Theoretically, blastocyst ET, which is more similar to the natural cycle than cleavage-stage ET, has a higher implantation potential. ${ }^{10}$ Previous studies have shown that decreased uterine contractility during the later luteal phase and the larger sizes of blastocysts would prevent the retrograde passage of embryos, which imply that blastocyst ET reduces the rate of ectopic pregnancy compared with cleavage-stage ET. ${ }^{11,12}$

These results support Chang and Suh's observation that transfer of three or more embryos with higher estimated embryo implantation potential was reported to be associated with an increased risk of ectopic pregnancy. ${ }^{4}$ Moreover, two studies suggested that the rate of ectopic pregnancy was not reduced after blastocyst ET compared with cleavage-stage ET. ${ }^{13}$

Here we report an interesting case of ruptured right tubal ectopic pregnancy post IVF who later on conceived spontaneously and delivered by caesarean section, a healthy male child.

\section{CASE REPORT}

Mrs X, a 32 year old female, primigravida, married life 21/2 years came in Emergency Department of Apollo Hospitals, Ahmedabad on 13 November 2013. She presented with complaints of amenorrhoea 2 months, last mentrual period (LMP)-12 September 2013. She had severe pain abdomen associated with 3 episodes of vomiting since night of 12 November 2013, uneasiness, difficulty in breathing and spotting vagina on and off since 29 October 2013. She had been evaluated for primary infertility and was diagnosed to have polycystic ovary syndrome (PCOS) in 2012. Diagnostic laparoscopy with ovarian drilling was done in August 2012. Uterus was normal and both tubes were patent with free spillage of dye seen. She did not have any other positive past medical history and family history. She had undergone IVF at ART centre. History of ovum pick up done on July 2013. First cycle of IVF done in August 2013 when 2 embryos (day 4 ), frozen thawed transferred but the cycle failed. Second cycle of IVF done in September 2013 when 3 embryos (day 4) frozen thawed transferred and resulted in positive pregnancy test on 13 October 2013 by serum beta human chorionic gonadotropin (hCG). She was on micronized progesterone and estradiol following the embryo transfer. She was given injection pubergen 5000 IU on 29 October 2013 and 02 November 2013 as she had history of spotting per vagina. Her serial serum beta hCG levels were as follows: $2031 \mathrm{mIU}$ on 29 October 2013, $8031 \mathrm{mIU}$ on 06 November 2013, 22,000 mIU on 12 November 2013, $10,990 \mathrm{mIU}$ on 13 November 13. Transvaginal ultrasound was done on 12 November 2013 and also thrice before in private clinics but it failed to show any intrauterine or extrauterine pregnancy.

\section{Obstetric history}

Primigravida, married life $-21 / 2$ years

\section{Menstrual history}

3-4/28-30 days, regular, normal flow and pain for 2 days.

Patient was admitted at Apollo hospitals on 13 November 2020 for further management.

\section{On examination}

Patient was well built, weight- $89 \mathrm{~kg}$, height- $170 \mathrm{~cm}$, body mass index (BMI)-30.8, pulse rate- $86 / \mathrm{min}$, blood pressure (BP)-126/65 $\mathrm{mm}$ of $\mathrm{Hg}$, temperature-98.2 $2^{0} \mathrm{~F}$, cardiovascular system (CVS)-S1S2 normal, respiratory rate (RS-RR)-22/min, and chest clear.

Per abdomen examination - soft, obesity+, distended, tenderness++ and more in suprapubic region.

Bimanual examination - cervix closed, elongated, cervical movement tender, uterus anteverted, anteflexed, tenderness++, more in right fornix, and left fornix clear.

Investigations - On 13 November 2013: hemoglobin ( $\mathrm{Hb})$ - $10.3 \mathrm{gm} \%$, white cell count $-9,170 / \mathrm{cu} \mathrm{mm}$, differential count (DC) - neutrophils (N) 82\%, lymphocytes (L) 15\%, monocytes (M) 2\%, eosinophils (E) 1\%, platelets2,19,000/cu mm, bleeding time-2 min, clotting time- 6 min, prothrombin time (PT)-12.1 $\mathrm{sec}$ (T), $12 \mathrm{sec}$ (C), international normalized ratio (INR)-1.01, serum creatinine- 0.7 , blood group-A positive, serum sodium-133 $\mathrm{mmol} / \mathrm{L}$, serum potassium-4.05 $\mathrm{mmol} / \mathrm{L}$, serum chloride$101 \mathrm{mmol} / \mathrm{L}$, human immunodeficiency virus (HIV) and surface antigen of the hepatitis B virus (HBsAg)nonreactive.

Ultrasound whole abdomen showed uterus measuring $8.9 \times 6.1 \times 5.1 \mathrm{~cm}$, endometrial thickness $-7 \mathrm{~mm}$, complex mixed echogenic lesion measuring $9.3 \times 5.8 \mathrm{~cm}$ in right adnexal region, $6.4 \times 4 \mathrm{~cm}$ seen in left adnexal region, both ovaries normal, free fluid in pelvis and right paracolic gutter suggestive of ruptured right tubal ectopic pregnancy.

A clinical diagnosis of ruptured right tubal ectopic pregnancy with haemoperitonium was made.

She underwent exploratory laparotomy followed by removal of right ectopic pregnancy, right salpingectomy and peritoneal lavage under general anesthesia (GA) on 13 November 2013 (Figure 1 and 2).

Intraoperative findings - haemoperitonium++, fresh blood with blood clots around $800 \mathrm{ml}$ in peritoneal cavity, in both paracolic gutter and under the diaphragm, uterus bulky, ruptured right tubal ectopic pregnancy at ampullary region 
with organized blood clot measuring $4.5 \times 4 \mathrm{~cm}$, left tube and both ovaries healthy (Figure 3 and 4 ).

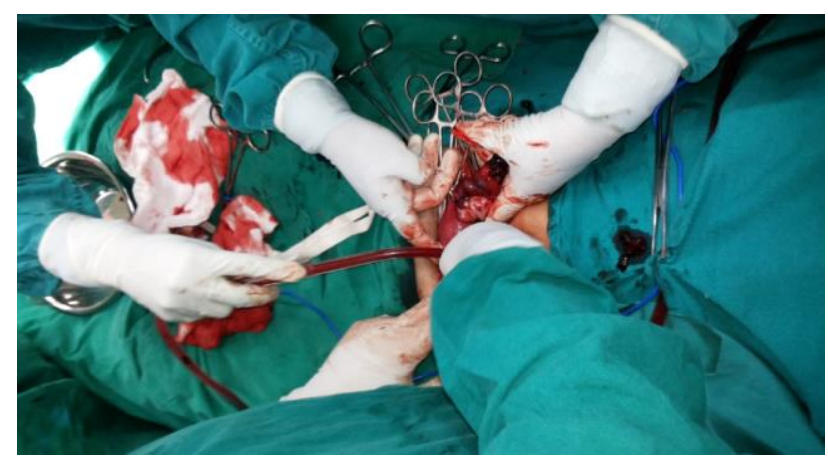

Figure 1: Exploratory laparotomy for right ruptured tubal ectopic pregnancy and peritoneal lavage.

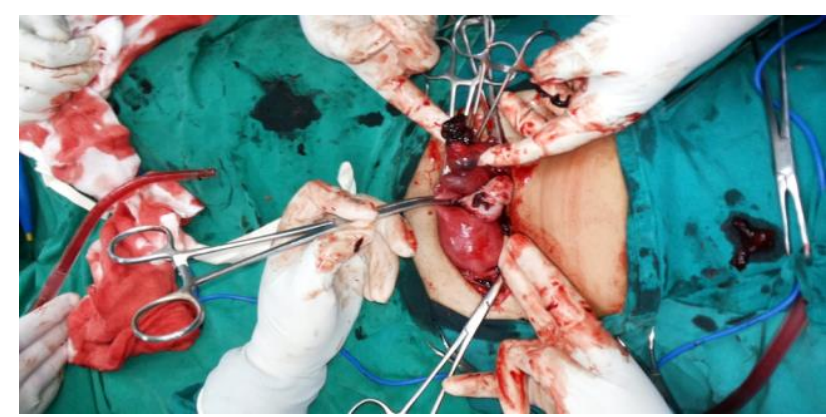

Figure 2: Ruptured ectopic pregnancy in ampullary part of right fallopian tube.

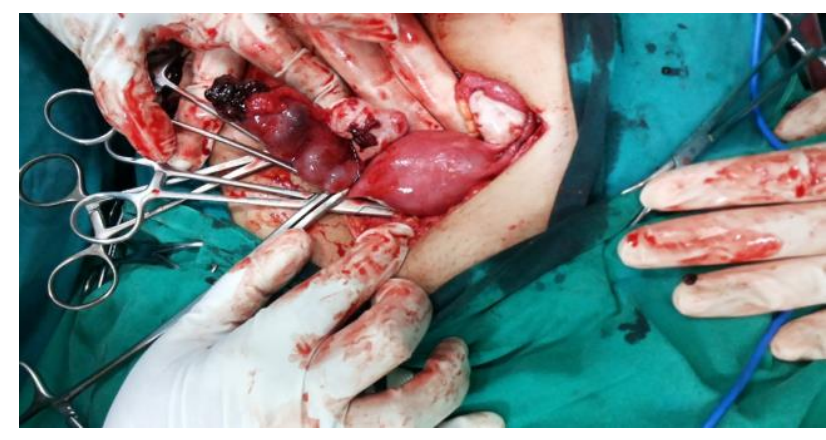

Figure 3: Ruptured right tubal ectopic pregnancy with organized blood clot measuring $4.5 \times 4 \mathrm{~cm}$ at fimbrial end.

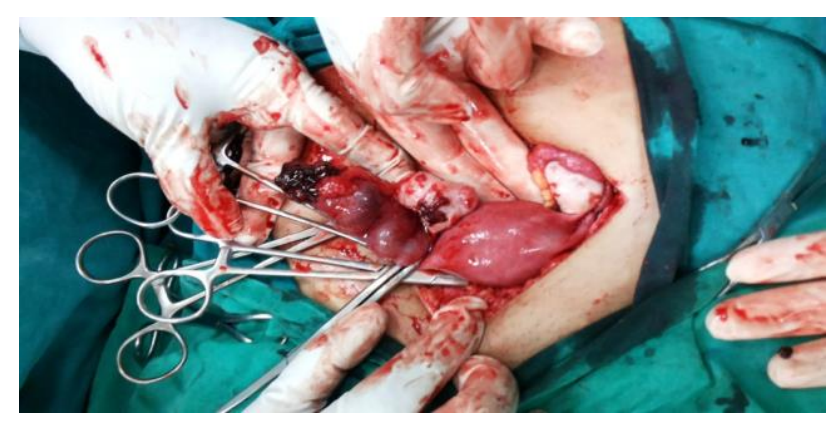

Figure 4: Uterus bulky, normal left tube and bilateral healthy ovaries.
Dialatation and curettage was done. One unit packed cell volume was transfused intraoperatively. Postoperative recovery was uneventful. Complete blood count (CBC) was done on 15 November 2013, hemoglobin (Hb) was $10.4 \mathrm{gm} \%$.

Histopathology report of right tube and right ectopic mass revealed features consistent with those of ectopic pregnancy.

Fearing that she might have repeat ectopic pregnancy, patient did not to go for IVF again. She did not take any treatment for next 3 months. She was advised lifestyle modification, weight reduction, strict diet control and regular exercises which she followed. She was on tablet metformin sustained release (SR) twice daily and she lost $6 \mathrm{~kg}$ weight in 6 months. She underwent 3 cycles of ovulation induction with clomiphene citrate and intrauterine insemination in one cycle. However she conceived spontaneously in her $4^{\text {th }}$ cycle without any treatment.

This pregnancy was eventful and a complicated one as she developed herpes simplex labialis in $24^{\text {th }}$ week of pregnancy with multiple painful eruptions around her lips, was given symptomatic treatment with local ointment and oral acyclovir for 5 days. The lesions subsided after 15 days. At 25 weeks she was admitted in Apollo Hospital on 25 January 2015 with high grade fever with chills, cold and cough and loss of appetite since 10 days, she was diagnosed to have pyrexia of unknown origin, was managed with intravenous (I/V) antibiotics and I/V fluids and discharged on 30 January 2015.

At 35 weeks of pregnancy, she was again admitted with preterm labour pain at Apollo Hospital on 09 April 2015. High vaginal swab and urine sent for culture and sensitivity. Urine culture report showed growth of $E$. Coli. Injection zostum $1.5 \mathrm{gm} \mathrm{I} / \mathrm{V} 12$ hourly given. Injection betamethasone $12 \mathrm{mg}$ intramuscular (IM), 2 doses given 12 hours apart for lung maturity. She went into active labour and as her cardiotocography (CTG) monitoring showed early decelerations, she was taken for emergency caesarean section under SA for foetal distress on 10 April 2015. A healthy male child, weight $2.44 \mathrm{~kg}$ delivered at 12.20 p.m. As baby's head was deeply engaged in pelvis, baby was delivered by Patwardhan's method. Her postoperative period in the hospital was uneventful. Baby developed neonatal hyperbilirubinemia on 4th day of birth, was admitted in neonatal intensive care unit (NICU) and given phototherapy for 2 days. She was discharged with healthy baby on 15 April 2015. On follow up after 4 weeks, patient was fine, had no complaints, caesarean scar was healthy. Her child was healthy, growing well, was taking breast feed properly.

\section{DISCUSSION}

Ectopic pregnancy is a dramatic life threatening event in a woman's reproductive life, especially after a long, 
expensive and difficult course of treatment for infertility. EP accounts for around 1-2\% of all natural conceptions, and this prevalence increases following assisted reproductive techniques, to range between $2.1 \%$ and $8.6 \%$ and it can reach up to $11 \%$ in women with tubal factors infertility history. ${ }^{3}$ EP occurs mostly in the fallopian tubes (90\%), and the most common region is the ampula. Its prompt diagnosis and appropriate management is important in preventing severe morbidity and mortality.

The first pregnancy obtained with IVF by Steptoe et al was ectopic, in a patient with history of tubal factor infertility. ${ }^{14}$ Theories regarding the genesis of spontaneous ectopic pregnancy are well known. Damage to the endosalpinx produced by sexually transmitted infections, distortion of pelvic anatomy caused by disease like endometriosis or by adhesions after previous surgical interventions, surgery performed in the fallopian tubes, mullerian malformations, hormone concentration imbalance during the menstrual cycle, late ovulation, history of a previous ectopic pregnancy. ${ }^{4}$ In our case there was no risk factor like history of pelvic inflammatory disease, endometriosis or previous pelvic surgeries but there was history of IVF-ET done.

Correct diagnosis of ectopic pregnancy is made on patient's history, clinical acumen, serum beta-hCG levels and pelvic ultrasound. Preoperative diagnosis is often difficult, challenging and unpredictable. In our case, the tubal ectopic was initially missed on transvaginal ultrasound done 4 times outside at private clinics. The rise in beta hCG in our case was not correlating with intrauterine pregnancy and moreover the beta hCG level dropped later on which gave a clue to ruptured ectopic pregnancy and it was confirmed by transvaginal ultrasound done at our hospital.

Rates of ectopic pregnancy following IVF-ET have been reduced by restricting the number of embryos transferred, avoiding deep fundal transfer and tubal embryo transfer (TET), injecting a smaller volume of fluid during ET, and transferring frozen-thawed embryos. ${ }^{3,4,15,16}$ The mechanism of post-ART ectopic pregnancies can originate from the direct transfer of embryo to the tube or migration of the embryo from the endometrial cavity to the tube.

In general, there has been a belief that higher progesterone concentrations in the luteal phase may reduce uterine contractility during fresh ET, which may prevent the embryo from migrating into the fallopian tubes. ${ }^{17}$ However, a growing number of studies are now available to shed some light on lower ectopic pregnancy rate following frozen-thawed ET.

There is some evidence that transferring blastocysts that are ready to implant instead of earlier embryos may also reduce the incidence. Sometimes, however, despite the best-laid plans, ectopic pregnancies do occur. ${ }^{18}$ In our case, 3 embryos (day 4) frozen thawed were transferred which were cleavage stage embryo, these factors could have led to ectopic pregnancy.
An aggravation of the ET technique might be considered as a risk factor for an ectopic pregnancy after IVF/intracytoplasmic sperm injection (ICSI) treatment, too. The use of a tenaculum might arouse uterine contractions especially in the subendometrial myometrium. Lesny et al reported a significant increase in uterine cervico-fundal (retrograde) contractility documented by transvaginal ultrasound after the insertion of a tenaculum. The increase of a retrograde contractility pattern should be regarded as another risk factor for an ectopic pregnancy besides the tubal status. ${ }^{19}$

This is supported by the observation by Fanchin et al that an increased frequency of subendometrial contractions during an aggravated ET procedure is significantly associated with reduced pregnancy rates and that myometrial contractions should be avoided by using a gentle, atraumatic transfer technique. In this study, cervico-fundal (retrograde) peristalsis prevails in $64 \%$, antegrade peristalsis was only observed in a minority of subjects. ${ }^{20}$ In our case aggravation of the ET procedure might be an important etiologic risk factor for the ectopic tubal pregnancy.

Infertility is a problem affecting $8-12 \%$ of couples worldwide. $^{21}$

The associations between infertility and EP are complex, as one of them could be simultaneously a cause and the other a consequence. ${ }^{22,23}$

There is an increased risk of developing EP following fertility treatment, which could be due to the effects of the treatment or the pre-existing disorder. ${ }^{4}$ In our case the patient had undergone IVF-ET for primary infertility, resulting into ruptured tubal ectopic pregnancy for which salpingectomy was done. Inspite of these factors which could have aggravated her infertility problem, she could spontaneously conceive.

Infertility is frequently met in PCOS patients, with a prevalence of $74 \%$, and in $40 \%$ of cases it is actually the motive of presentation to the doctor. ${ }^{24,25}$ Infertility is the consequence of chronic anovulation, being usually accompanied by menstrual abnormalities: dysfunctional uterine bleeding, oligomenhorrea, and amenorrhea. The presence of regular menstrual bleeding doesn't exclude anovulation. Twenty one per cent of the patients with hyperandrogenism have regular menstrual cycles that are anovulatory. ${ }^{26}$ In our case the patient was obese, a known case of PCOS had regular menstrual cycles with anovulation who presented with infertility.

Weight loss has been studied by many authors, all of them reporting the improvement of hyperinsulinemia and hyperandrogenism, simultaneously with the recovery of normal menstrual cycles and the appearance of spontaneous pregnancies in $30 \%$ of the patients. ${ }^{27}$ These fortunate effects were observed even with a modest weight loss of $2-5 \% .{ }^{28}$ In this case, our patient had $5 \%$ weight loss 
which helped her to conceive. Metformin is an insulin sensitizer drug given in PCOS patients.

Administered to the PCOS patients, metformin may improve the menstrual disorders and anovulation in 50\% of the studied patients, with increased probability of response in the patients with hyperinsulinemia, less severe menstrual disorders and a lower level of free testosterone. ${ }^{29}$ Thus, it has been speculated upon the fact that metformin is efficient in the category of patients in which the insulin resistance has an important role in pathogenesis. In our case patient was given metformin SR twice daily which helped in reducing insulin resistance and thus in spontaneous conception.

In review of literature we could not find any case report where spontaneous pregnancy resulted following post IVF ruptured ectopic pregnancy. Thus lifestyle modification, reassurance, weight reduction, healthy diet for PCOS, reduction of insulin resistance and correction of hormonal imbalance might be a crucial factor in the successful outcome.

\section{CONCLUSION}

Diagnosis of ruptured tubal ectopic pregnancy is made based on patient's history, clinical acumen, serum beta hCG levels and pelvic ultrasound. EP must be suspected in patients presenting with abdominal pain after IVF treatment despite low serum beta hCG levels. A high index of suspicion in women is needed, early and timely diagnosis and management with laparotomy or laparoscopy can result in favourable outcome. This case highlights the fact that patient who underwent IVF treatment resulting in ruptured tubal ectopic pregnancy can have spontaneous conception and a successful obstetric outcome.

\section{Funding: No funding sources}

Conflict of interest: None declared

Ethical approval: Not required

\section{REFERENCES}

1. Khan KS, Wojdyla D, Say L, Gulmenzoglu AM, van Look PF. WHO analysis of causes of maternal death: a systematic review. Lancet. 2006;367:1066-74.

2. Tal S, Einat PS, Eylon L, Ofer F, Adrian E. Unusual case of recurrent heterotopic pregnancy after bilateral salpingectomy and literature review. RBM Online. 2013;26:59-61.

3. Clayton HB, Schieve LA, Peterson HB, Jamieson DJ, Reynolds MA, Wright VC. Ectopic pregnancy risk with assisted reproductive technology procedures. Obstet Gynecol. 2006;107:595-604.

4. Chang HJ, Suh CS. Ectopic pregnancy after assisted reproductive technology: what are the risk factors? Curr Opin Obstet Gynecol. 2010;22:202-7.

5. Nazari A, Askari HA, Check JH, O'Shaughnessy A. Embryo transfer technique as a cause of ectopic pregnancy in in vitro fertilization. Fertil Steril. 1993;60:919-21.

6. Li W, Wang G, Lin T, Sun W. Misdiagnosis of bilateral tubal pregnancy: a case report. J Med Case Rep. 2014;8:342.

7. Jena SK, Singh S, Nayak M, Das L, Senapati S. Bilateral simultaneous tubal ectopic pregnancy: a case report, review of literature and a proposed management algorithm. J Clin Diagn Res. 2016;10(3):QD01-3.

8. Hortu I, Akman L, Akdemir A, Ergenoglu M, Yeniel $\mathrm{O}$, Sendag F. Management of ectopic pregnancy in unusual locations: five-year experience in a single center. J Clin Exp Invest. 2017;8(3):90-5.

9. Sugawara N, Sato R, Kato M, Manome T, Kimura Y, Araki $\mathrm{Y}$, et al. Bilateral tubal pregnancies after a single-embryo transfer. Reprod Med Biol. 2017;16(4):396-400.

10. Mains L, Van Voorhis BJ. Optimizing the technique of embryo transfer. Fertil Steril. 2010;94:785-90.

11. Fanchin R, Ayoubi JM, Righini C, Olivennes F, Schonauer LM, Frydman R. Uterine contractility decreases at the time of blastocyst transfers. Hum Reprod. 2001;16:1115-9.

12. Schoolcraft WB, Surrey ES, Gardner DK. Embryo transfer: techniques and variables affecting success. Fertil Steril. 2001;76:863-70.

13. Milki AA, Jun SH. Ectopic pregnancy rates with day 3 versus day 5 embryo transfer: a retrospective analysis. BMC Pregnancy Childbirth. 2003;3:7.

14. Steptoe PC, Edwards RG. Reimplantation of a human embryo with subsequent tubal pregnancy. Lancet. 1976;1:880-2.

15. Pope CS, Cook EK, Arny M, Novak A, Grow DR. Influence of embryo transfer depth on in vitro fertilization and embryo transfer outcomes. Fertil Steril. 2004;81:51-8.

16. Ishihara O, Kuwahara A, Saitoh H. Frozen-thawed blastocyst transfer reduces ectopic pregnancy risk: an analysis of single embryo transfer cycles in Japan. Fertil Steril. 2011;95:1966-9.

17. Shapiro BS, Daneshmand ST, De Leon L, Garner FC, Aguirre M, Hudson C. Frozen-thawed embryo transfer is associated with a significantly reduced incidence of ectopic pregnancy. Fertil Steril. 2012;98:1490-4.

18. Andres MP, Campillos, Lapresta, Lahoz, Crespo R, Tobajas J. Management of ectopic pregnancies with poor prognosis through USG guided intrasacular injection of methotrexate, series of 14 cases. Arch Gynecol Obstet. 2012;285:529-33.

19. Lesny P, Killick SR, Robinson J, Raven G, Maguiness SD. Junctional zone contractions and embryo transfer: is it safe to use a tenaculum? Hum Reprod. 1999; 14:2367-70.

20. Fanchin R, Righini C, Olivennes F, Taylor S, de Ziegler D, Frydman R. Uterine contractions at the time of embryo transfer alter pregnancy rates after invitro fertilization. Hum Reprod. 1998;13:1968-74. 
21. Mascarenhas MN, Flaxman SR, Boerma T, Vanderpoel S, Stevens GA. National, regional, and global trends in infertility prevalence since 1990: a systematic analysis of 277 health surveys. PLoS Med. 2012;9(12):1001356.

22. Jurkovic D, Wilkinson H. Diagnosis and management of ectopic pregnancy. BMJ. 2011;342:d3397.

23. Sivalingam VN, Duncan WC, Kirk E, Shephard LA, Horne AW. Diagnosis and management of ectopic pregnancy. J Fam Plann Reprod Health Care. 2011;37:231-40.

24. Goldzieher JW, Green JA. Clinical and biochemical features of polycystic ovarian disease. Fertil Steril. 1963; 14:631-53.

25. Franks S. Polycystic ovary syndrome. N Engl J Med. 1989;333:853-61.

26. Carmina E, Lobo RA. Do hyperandrogenic women with normal menses have polycystic ovary syndrome? Fertil Steril. 1999;71:319-22.

27. Pasquali R, Antenucci D, Casimirri F. Clinical and hormonal characteristics of obese amenorrheic hyperandrogenic women before and after weight loss. J Clin Endocrinol Metab. 1989;68:173-9.

28. Huber-Bucholz MM, Carey DGP, Norman RJ. Restoration of reproductive potential by lifestyle modification in obese polycystic ovary syndrome. J Clin Endocrinol Metab. 1999;84:1470-4.

29. Moghetti P, Castello R, Negri C, Tosi F, Perrone F, Caputo M, Zanolin E, Muggeo M. Metformin effects on clinical features, endocrine and metabolic profiles, and insulin sensitivity in polycystic ovary syndrome: a randomized, double-blind, placebo-controlled 6month trial, followed by open, long-term clinical evaluation. J Clin Endocrinol Metab. 2000;85:139-46.

Cite this article as: Nahar K, Nahar N. Spontaneous pregnancy following post in vitro fertilization ruptured tubal ectopic pregnancy. Int J Reprod Contracept Obstet Gynecol 2020;9:4738-43. 